\section{Impact of bone marrow fibrosis grade in post- polycythemia vera and post- essential thrombocythemia myelofibrosis: A study of the MYSEC group}

To the Editor:

Polycythemia vera (PV) and essential thrombocythemia (ET) are myeloproliferative neoplasms that can progress to post-PV (PPV-) myelofibrosis (MF) and post-ET (PET-) MF, also known as secondary myelofibrosis (SMF). The 2008 diagnostic criteria for SMF require the presence of significant bone marrow fibrosis (BMF). The impact of BMF grading on presentation and outcome in primary MF (PMF) has been extensively explored, ${ }^{1-3}$ while only limited information is available in SMF. ${ }^{4}$

For this reason, we analyzed the MYSEC (MYelofibrosis SECondary to $P V$ and ET) cohort, recently updated, now including 805 SMF patients among 17 centers in Europe and United States. ${ }^{5}$

The SMF diagnoses were performed between 1981 and 2017. Particular attention was given in order to avoid the inclusion of pre-fibrotic MF. BMF at time of SMF evolution was semi-quantitatively defined as grade 2 (BMF2) in case of a diffuse increase in reticulin with extensive intersections, occasional bundles of collagen and/or osteosclerosis, or as grade 3 (BMF3) if presenting with coarse bundles of collagen, often associated with osteosclerosis. Chi-square or Fisher exact test for categorical variables and Wilcoxon rank-sum test for continuous ones were applied for exploring patterns of association between BMF grading and patients' characteristics. Multiple logistic regression analysis was used for the same purpose, and adjusted odds ratio together with $95 \%$ Confidence Interval $(95 \% \mathrm{Cl})$ were calculated. Rates of events were evaluated with a Poisson regression model. Survival curves were estimated using the Kaplan-Meier method and log-rank test was calculated. A Cox proportional hazard regression model was applied for multivariate survival analysis. The MYSEC study was approved by the Review Board of each Institution, and conducted in accordance with the Declaration of Helsinki.

Detailed information on BMF grading was available in 675 MYSEC patients: BMF2 was reported in 443 (65.6\%) and BMF3 in 232 (34.4\%). At SMF diagnosis, we did not find any correlation between BMF grade and age, gender, time to progression from PV/ET, spleen and liver size, presence of constitutional symptoms and marrow blast percentage (Table S1). No relationship was noticed with abnormal karyotype (stratified as previously reported) $^{6}$ with data available in about $50 \%$ of the cohort (Table S1). Looking at genotype, BMF grading was not differently distributed among the three driver mutations as well as in "triple negative" cases (Table S1).

On the contrary, in univariate analysis, there was a significant association between BMF3 and previous diagnosis of ET $(P=.02)$, decreased hemoglobin levels $(P<.0001)$, reduced platelet $(P<.0001)$ and leukocyte counts $(P=.001)$, higher percentage of circulating blasts $(P=.01)$ and $\mathrm{LDH}$ value $(P=.001)$ (Table S1). In multivariate analysis, BMF3 resulted significantly correlated with previous ET $(P=.006)$, lower hemoglobin level $(P=.0002)$ and reduced platelet count $(P=.0006)$ (Table S2). Patients with BMF2 were more frequently included in the lower MYSEC-PM (MYSEC-Prognostic Model) risk groups, while those with BMF3 were enriched in the higher risk categories $(P<.0001$, Table $S 1)$

Median follow-up of the cohort was 11.0 (range, 0.6-41.4) years from ET/PV phase to SMF and 3.1 (range, 0.6-27) years after SMF transformation. More patients with BMF2 received cytoreductive therapies during the course of SMF $(P<.0001$; Table S1). On the contrary, more patients with BMF3 underwent allogenic stem cells transplantation $(P<.0001$, Table S1).

No differences were noticed in terms of cumulative incidence of thrombosis between BMF subgroups (Table S3). Evolution into postSMF blast phase was documented in 62 (9.2\%) patients, without any effect of BMF grade (Table S3).

We found a significantly higher mortality in case of BMF3 vs BMF2 $(P<.0001$, Table S3). Median survival was 8.2 (95\% Cl: 7.7-11.8) years in the BMF2 subgroup, while it corresponded to 7.4 (95\% Cl: 5.1-10.1) years for BMF3 ( $P=.011$, Figure 1$)$. Nevertheless, when adjusting theimpact of BMF3 grade on survival in a Cox multivariate analysis that considered MYSEC-PM categories (low and intermediate-1 vs intermediate- 2 and high), the BMF grade lost its prognostic relevance $(P=.13)$.

Our study provides evidence that BMF grade is associated with a specific phenotype in SMF. Multivariate analysis showed that BMF3 clustered with lower hemoglobin and reduced platelet values. Similarly, recent papers on PMF demonstrated that blood count counts are inversely correlated with BMF grade.,3 In our cohort of 675 SMF patients, BMF3 was significantly associated with PET- vs PPV-MF, irrespectively of previous ET/PV duration. Another study on 755 PMF and 344 SMF reported that "advanced" BMF grades $(2,3)$ are more frequent in PPV-MF than in PET-MF or PMF. ${ }^{4}$ This result could be explained by the fact that even cases with BMF grade lower than 2 were retrospectively included among those $344 \mathrm{SMF} .{ }^{4}$ In our dataset, BMF3 clustered with intermediate-2 and high MYSEC-PM categories and detrimental SMF outcome. Likewise, higher IPSS (International Prognostic Scoring System) subgroups were enriched by PMF cases with a more advanced BMF, and a consequently worse survival. ${ }^{3}$ 


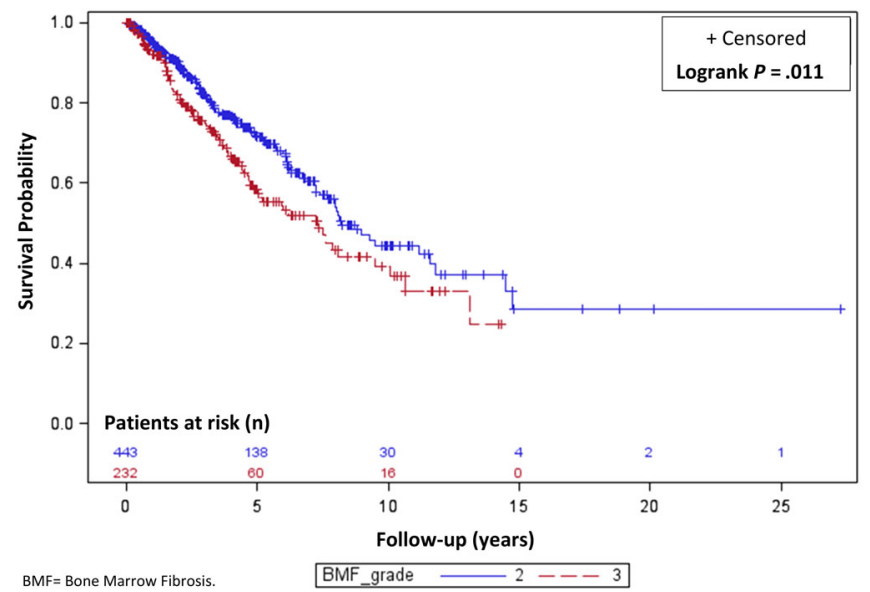

FIGURE 1 Kaplan-Meier survival estimate of patients with postpolycythemia vera and post-essential thrombocythemia myelofibrosis according to the bone marrow fibrosis grade

In our SMF cohort, BMF3 resulted associated with a significant lower use of cytoreductive therapies and a higher rate of allogenic stem cells transplantation. It might be plausible that the less myeloproliferative phenotype and the expected worse outcome of these patients had influenced physicians' treatment decision.

Available information indicates that BMF grade has a different impact on PMF- vs SMF-specific prognostic scoring systems. In PMF, BMF grading provided additional information to IPSS and was recently included in the MIPSS70 (Mutation-Enhanced IPSS for patients aged less than 70 years) score. ${ }^{2,3}$ Differently, BMF did not implement SMF survival prediction as defined by the MYSEC-PM. ${ }^{5}$ It will be of interest to see if BMF3 clusters with "unfavorable" additional mutations in myeloid genes, as pointed out in a study on $\mathrm{PMF}^{3}$

In conclusion, the development of progressive anemia and thrombocytopenia during the course of PV and ET, besides suggesting an evolution into SMF, might be also a surrogate of BMF3 grade, eventually resulting in a worse outcome. This suggests to monitor carefully cell blood count in PV and ET patients, with the aim to detect early a possible transformation into SMF.

\section{FUNDING INFORMATION}

The Varese group was funded by the Fondazione Regionale Ricerca Biomedica, Milan, Italy [FRRB project no. 2015-0042, Genomic profiling of rare hematologic malignancies, development of personalized medicine strategies, and their implementation into the Rete Ematologica Lombarda (REL) clinical network], by the Fondazione Matarelli (Milano, Italy), the Fondazione Rusconi (Milano, Italy) and AIL Varese ONLUS. PG and AMV were supported by AIRC $5 \times 1000$ called "Metastatic disease: the key unmet need in oncology" to MYNERVA project, \#21267 (MYeloid NEoplasms Research Venture AIRC). RTS was supported in part by the Cancer Research and Treatment Fund, Inc., New York, NY.

\section{ORCID}

Barbara Mora (D) https://orcid.org/0000-0002-6325-3916

Paola Guglielmelli iD https://orcid.org/0000-0003-1809-284X

Elisa Rumi (D) https://orcid.org/0000-0002-7572-9504

Rami S. Komrokji (D) https://orcid.org/0000-0002-1876-5269

Jean-Jacques Kiladjian (D) https://orcid.org/0000-0002-8121-438X

Alessandra lurlo (D) https://orcid.org/0000-0002-4401-0812

Timothy Devos (D) https://orcid.org/0000-0002-6881-417X

Francesca Palandri (D) https://orcid.org/0000-0001-8367-5668

Valerio De Stefano (D) https://orcid.org/0000-0002-5178-5827

Marco Ruggeri (D) https://orcid.org/0000-0003-1557-0957

Francesco Albano (D) https://orcid.org/0000-0001-7926-6052

Giulia Benevolo (D) https://orcid.org/0000-0002-3200-9654

Silvia Uccella (D) https://orcid.org/0000-0002-5714-7295

Michele Merli (D) https://orcid.org/0000-0002-0905-5927

Tiziano Barbui (D) https://orcid.org/0000-0003-2747-6327

Mario Cazzola (D) https://orcid.org/0000-0001-6984-8817

Alessandro M. Vannucchi (D) https://orcid.org/0000-0001-5755-0730

Francesco Passamonti (D) https://orcid.org/0000-0001-8068-5289

Barbara Mora ${ }^{1}$ (D), Paola Guglielmelli ${ }^{2}$ (D), Elisa Rumi ${ }^{3}$ (D), Margherita Maffioli ${ }^{1}$, Daniela Barraco ${ }^{1}$, Alessandro Rambaldi ${ }^{4}$, Marianna Caramella ${ }^{5}$, Rami S. Komrokji ${ }^{6}$ (D) JeanJacques Kiladjian ${ }^{7}$ (D), Jason Gotlib ${ }^{8}$, Alessandra lurlo ${ }^{9}$ (D), Francisco Cervantes ${ }^{10}$, Timothy Devos ${ }^{11}$ (D), Francesca Palandri ${ }^{12}$ (D), Valerio De Stefano ${ }^{13}$ (D), Marco Ruggeri ${ }^{14}$ (D), Richard T. Silver ${ }^{15}$, Francesco Albano ${ }^{16}$ (D), Giulia Benevolo ${ }^{17}$ (iD), Chiara Cavalloni ${ }^{3}$, Silvia Uccella ${ }^{18}$ (D), Raffaella Accetta ${ }^{19}$, Claudia Siracusa ${ }^{19}$, Stefania Agnoli ${ }^{1}$, Michele Merli ${ }^{1}$ (ID), Tiziano Barbui ${ }^{20}$ (D), Lorenza Bertù $^{21}$, Mario Cazzola ${ }^{3}$ (D), Alessandro M. Vannucchi ${ }^{2}$ (D), Francesco Passamonti ${ }^{1,21}$ (D)

${ }^{1}$ Hematology, Ospedale di Circolo, ASST Sette Laghi, Varese, Italy ${ }^{2}$ CRIMM; Center Research and Innovation of Myeloproliferative Neoplasms, University of Florence, Florence, Italy

${ }^{3}$ Hematology, Department of Molecular Medicine, Fondazione IRCCS Policlinico San Matteo, University of Pavia, Pavia, Italy

${ }^{4}$ Hematology and Bone Marrow Transplant Unit, ASST Papa Giovanni XXIII, Bergamo, Italy

${ }^{5}$ Hematology, ASST Grande Ospedale Metropolitano Niguarda, Milan, Italy ${ }^{6}$ Malignant Hematology Department, Blood and Marrow Transplantation, H. Lee Moffitt Cancer Center and Research Institute, Tampa, Florida

${ }^{7}$ Hôpital Saint-Louis et Université Paris Diderot, Paris, France

${ }^{8}$ Divisions of Hematology, Stanford Cancer Institute, Stanford University School of Medicine, Stanford, California

${ }^{9}$ UOC Oncoematologia, Fondazione IRCCS Ca' Granda Ospedale Maggiore Policlinico, Milan, Italy

${ }^{10}$ Hematology Department, Hospital Clinic, IDIBAPS, University of Barcelona, Barcelona, Spain

${ }^{11}$ Department of Hematology, University Hospitals Leuven (UZ Leuven), Leuven, Belgium 
${ }^{12}$ Institute of Hematology, Azienda Ospedaliero-Universitaria S.Orsola-Malpighi di Bologna, Bologna, Italy

${ }^{13}$ Hematology, Fondazione Policlinico Universitario A. Gemelli IRCCS, Università Cattolica del Sacro Cuore, Rome, Italy

${ }^{14}$ Hematology Department, San Bortolo Hospital, Vicenza, Italy

${ }^{15}$ Richard T. Silver Myeloproliferative Neoplasms Center, Weill Cornell Medical Center, New York, New York

${ }^{16}$ Hematology - Dept. of Emergency and Organ Transplantation, University of Bari, Bari, Italy

${ }^{17}$ Struttura Complessa (S.C.) di Ematologia, Presidio Molinette Azienda Universitario-Ospedaliera (A.O.U) Città della Salute e della Scienza di Torino, Torino, Italy

${ }^{18}$ Department of Surgical and Morphological Sciences, Ospedale di Circolo e Fondazione Macchi, ASST Sette Laghi, University of Insubria,

Varese, Italy

${ }^{19}$ Laboratory of Medicine, Service of cytogenetics and medical genetics, Ospedale di Circolo, ASST Sette Laghi, Varese, Italy

${ }^{20}$ FROM Research Foundation, ASST Papa Giovanni XXIII, Bergamo, Italy

${ }^{21}$ Department of Medicine and Surgery, University of Insubria, Varese, Italy

Correspondence

Francesco Passamonti, Hematology, University Hospital "Ospedale di Circolo e Fondazione Macchi - ASST Sette Laghi", University of Insubria, Viale L. Borri 57, 21100, Varese, Italy. Email: francesco.passamonti@uninsubria.it

\section{REFERENCES}

1. Thiele J, Kvasnicka HM. Grade of bone marrow fibrosis is associated with relevant hematological findings-a clinicopathological study on 865 patients with chronic idiopathic myelofibrosis. Ann Hematol. 2006; 85(4):226-232.

2. Guglielmelli P, Lasho TL, Rotunno G, et al. MIPSS70: mutation-enhanced International prognostic score system for transplantation-age patients with primary myelofibrosis. J Clin Oncol. 2018;36(4):310-318.

3. Guglielmelli P, Rotunno G, Pacilli A, et al. Prognostic impact of bone marrow fibrosis in primary myelofibrosis. A study of the AGIMM group on 490 patients. Am J Hematol. 2016;91(9):918-922.

4. Masarova L, Bose $P$, Daver $N$, et al. Patients with post-essential thrombocythemia and post-polycythemia vera differ from patients with primary myelofibrosis. Leuk Res. 2017;59:110-116.

5. Passamonti F, Giorgino T, Mora B, et al. A clinical-molecular prognostic model to predict survival in patients with post polycythemia vera and post essential thrombocythemia myelofibrosis. Leukemia. 2017;31(12): 2726-2731.

6. Mora B, Giorgino T, Guglielmelli P, et al. Value of cytogenetic abnormalities in post-polycythemia vera and post-essential thrombocythemia myelofibrosis: a study of the MYSEC project. Haematologica. 2018;103(9):e392-e394.

\section{SUPPORTING INFORMATION}

Additional supporting information may be found online in the Supporting Information section at the end of this article.
Received: 22 September 2019 Accepted: 30 September 2019

DOI: 10.1002/ajh.25651

Bone marrow blast elimination by the fifth day of $7+3$ induction is the strongest predictor of potential cure in patients with acute myeloid leukemia younger than 61 years of age: A long-term follow-up of a multi-center prospective study

To the Editor:

Multiple clinical and genomic factors have been shown to be associated with improved prognosis in acute myeloid leukemia (AML). Nevertheless, based on the recent European LeukemiaNet (ELN) guidelines, incorporating these features ${ }^{1}$ a significant portion of younger patients presenting with de-novo AML are still categorized in the intermediate-risk group. Refined precision of the outcome prediction is essential for the assignment of such patients to treatment, particularly allogeneic stem cell transplantation (allo-SCT).

Rapid eradication of blasts from the peripheral blood during induction therapy for $A M L$ is associated with a good response to initial treatment. ${ }^{2-4}$ Our previously conducted prospective observational study has aimed to identify the subgroup of leukemic patients in whom a prompt response to therapy would be a surrogate for a superior outcome. The findings of the study have demonstrated that early bone marrow (BM) response, defined as reduction in the number of BM blasts to less than $5 \%$ of BM cells by the fifth day of induction is a strong predictor of remission and improved $\mathrm{OS}^{5}$ in patients younger than 61 years old and/or those with non-adverse cytogenetics, receiving the $3+7$ induction regimen.

We herein report the results of prolonged prospective follow-up of a median of 64 months (range 17.5-96) demonstrating that among AML patients younger than 61 years of age, clearance of BM blasts to less than $5 \%$ by the fifth day of induction is not only the strongest predictor of good prognosis, but is also a promising marker for cure. Notably, our 